\title{
Toda systems in closed string tachyon condensation
}

\author{
Sunggeun Lee and Sang-Jin Sin \\ Department of Physics, Hanyang University, 133-791, Seoul
}

\begin{abstract}
We consider $t t^{*}$ equations appearing in the study of localized tachyon condensations. They are described by various Toda system when we consider the condensation by the lowest tachyon corresponding to the monomial $x y$. The tachyon potential is calculated as a solution to these equations. The Toda system appearing in the deformation of $\mathbb{C}^{2} / \mathbb{Z}_{n}$ by $x y$ is identical to that of $D_{n}$ singularity deformed by $x$. For $\mathbb{C}^{3} / \mathbb{Z}_{n}$ with $x y z$ deformation, we find only generic non-simple form, similar to the case appearing in $\mathbb{C} / \mathbb{Z}_{5} \rightarrow \mathbb{C} / \mathbb{Z}_{3}$ and we discuss the difficulties in these cases.
\end{abstract}




\section{Contents}

1. Introduction

2. Landau-Ginzburg description of orbifold geometry and $t t^{*}$ equation 2

3. $t t^{*}$ equations for $\mathbb{C}^{1} / \mathbb{Z}_{n}$

3.1 Deformation by $x$ : condensation of the most tachyonic state

3.2 Deformation by $x^{2}$

4. $t t^{*}$ equations for $\mathbb{C}^{2} / \mathbb{Z}_{n}$

4.1 General aspects of LG model without orbifold action 8

4.2 Orbifolded LG: the mirror of $\mathbb{C}^{2} / \mathbb{Z}_{n}$

5. Discussion 11

A. $\mathbb{C}^{3} / \mathbb{Z}_{n}$

A.1 $t t^{*}$ euqation for $\mathbb{C}^{3} / \mathbb{Z}_{5} \rightarrow \mathbb{C}^{3} / \mathbb{Z}_{3}$

A.2 $t t^{*}$ euqation for $\mathbb{C} / \mathbb{Z}_{5} \rightarrow \mathbb{C} / \mathbb{Z}_{3}$

B. A sample residue calculation 18

\section{Introduction}

The study of localized tachyon condensation [1, 2, 3, 4, 5] has been considered with many interesting developments. The basic picture is that tachyon condensation induces cascade of decays of the orbifolds to less singular ones until the spacetime supersymmetry is restored. Therefore the localized tachyon condensation has a geometric description as the resolution of the spacetime singularities.

Following the line of Vafa's reformulation of the problem in terms of Mirror LandauGinzburg theory, we worked out the detailed analysis on the fate of spectrum and the background geometry under the tachyon condensation as well as the question of what is the analogue of c-theorem with the GSO-projection in a series of papers $\llbracket$, 8, 9.

The study of tachyon dynamics would be greatly improved if we know the potential that governs the condensation process. In this direction, Dabholkar and Vafa 
the tachyon potential is given by the maximal charge and worked out the case describing the decay of $\mathbb{C}^{1} / \mathbb{Z}_{3}$ to $\mathbb{C}$. In the previous paper [10], we generalized the result to $\mathbb{C}^{2} / \mathbb{Z}_{n}$ for $n=3,4,5$. We encountered solvable non-linear equations called Painleve III as well as its degenerate case. So it is interesting to ask which integrable system is waiting us for general $n$ 's, if they are still integrable at all.

In this paper, we consider $\mathbb{C}^{2} / \mathbb{Z}_{n}$ as well as to $\mathbb{C}^{1} / \mathbb{Z}_{n}$ for any $n$ and show that the resulting systems are various Toda systems when we consider the condensation by the lowest tachyon corresponding to the monomial $x y$. We find that the potential is calculated as a solution to Toda equations. One interesting notice is that the Toda system appearing as $t t^{*}$ equation in the deformation of $\mathbb{C}^{2} / Z_{n}$ by $x y$ is identical to that of $D_{n}$ singularity by $x$, whose mirror dual geometry is not clear.

The outline of the paper is as follows. In section 2, we summarize the basic concepts to introduce languages. In section 3 , we consider the $t t^{*}$ equations for $\mathbb{C}^{1} / \mathbb{Z}_{n}$. In section 4 , we calculate the $t t^{*}$ equations for $\mathbb{C}^{2} / \mathbb{Z}_{n}$. In section 5 , we discuss and conclude. In appendix A, we calculate for $\mathbb{C}^{3} / \mathbb{Z}_{n} \rightarrow \mathbb{C}^{3}$ as well as for $\mathbb{C} \mathbb{Z}_{5} \rightarrow \mathbb{C} / \mathbb{Z}_{3}$ and discuss the difficulties in analyzing the resulting equations quantitatively. In appendix $\mathrm{B}$, we give a sample residue calculation for topological metric that is an important ingredient of $t t^{*}$ equations.

\section{Landau-Ginzburg description of orbifold geometry and $t t^{*}$ equation}

The Mirror of $\mathbb{C}^{2} / \mathbb{Z}_{n}$ is an orbifolded Landau-Ginzburg (LG) model with potential $W=$ $x^{n}+y^{n}$ 12, 13. The main steps for the tachyon potential is to calculate the $t t^{*}$ equation and their solutions. In this section we summarize the basic concepts on these material following [2] and [14].

Landau-Ginzburg description of $\mathbb{C}^{2} / \mathbb{Z}_{n}$ : Here we give a brief summary of LandauGinzburg formulation of localized tachyon condensation [2]. For simplicity we take $\mathbb{C}^{2} / \mathbb{Z}_{n}$. The orbifold $\mathbb{C}^{2} / \mathbb{Z}_{n}$ is defined by the $\mathbb{Z}_{n}$ action given by equivalence relation

$$
\left(X_{1}, X_{2}\right) \sim\left(\omega^{k_{1}} X_{1}, \omega^{k_{2}} X_{2}\right), \quad \omega=e^{2 \pi i / n} .
$$

We call $\left(k_{1}, k_{2}\right)$ as the generator of the $\mathbb{Z}_{n}$ action. The orbifold can be imbedded into the gauged linear sigma model(GLSM) [13]. The vacuum manifold of the latter is described by the D-term constraints

$$
-n\left|X_{0}\right|^{2}+k_{1}\left|X_{1}\right|^{2}+k_{2}\left|X_{2}\right|^{2}=t
$$

Its $t \rightarrow-\infty$ limit corresponds to the orbifold and the $t \rightarrow \infty$ limit is the $O(-n)$ bundle over the weighted projected space $W P_{k_{1}, k_{2}}$. In the latter case, $X_{0}$ direction corresponds to the non-compact fiber of this bundle and $t$ plays role of size of the $W P_{k_{1}, k_{2}}$. 
By dualizing this GLSM, we get a LG model with a superpotential 12]

$$
W=\exp \left(-Y_{1}\right)+\exp \left(-Y_{2}\right)+\exp \left(-Y_{0}\right)
$$

where twisted chiral fields $Y_{i}$ are periodic $Y_{i} \sim Y_{i}+2 \pi i$ and related to $X_{i}$ by $\operatorname{Re}\left[Y_{i}\right]=\left|X_{i}\right|^{2}$. Introducing the variable $u_{i}:=e^{-Y_{i} / n}$, the periodicity of $Y_{i}$ imposes the identification $: u_{i} \sim$ $e^{2 \pi i / n} u_{i}$ which necessitate modding out each $u_{i}$ by $\mathbb{Z}_{n}$. The D-term constraint is expressed as $e^{-Y_{0}}=e^{t / n} u_{1}^{k_{1}} u_{2}^{k_{2}}$ whose invariance requires that only one $\mathbb{Z}_{n}$ can be independent. The result is usually described by

$$
\left[W=u_{1}^{n}+u_{2}^{n}+e^{t / n} u_{1}^{k_{1}} u_{2}^{k_{2}}\right] / / \mathbb{Z}_{n}
$$

which describe the mirror Landau-Ginzburg model of the linear sigma model. As $t \rightarrow-\infty$ limit, mirror of the orbifold is

$$
\left[W=u_{1}^{n}+u_{2}^{n}\right] / / \mathbb{Z}_{n}
$$

¿From now on we use $x, y$ instead of $u_{1}, u_{2}$ for notational convenience and fix $\left(k_{1}, k_{2}\right)=$ $(1, k)$ since it is allowed [8]. Since it is not ordinary Landau-Ginzburg theory but an orbifolded version, the chiral ring structure of the theory is slightly different from that of LG model. For example, the chiral ring of LG model is

$$
\left\{x^{i} y^{j} \mid i, j=1,2, \cdots, n-1\right\}
$$

while that of orbifolded LG model is

$$
\left\{x^{i} y^{j} \mid(i, j)=l(1, k), l=1,2, \cdots, n-1\right\}
$$

where $(1, k)$ is the generator of the chiral ring and $k$ defines the orbifold action in $\mathbb{C}^{2}$ :

$$
X_{1} \rightarrow \omega X_{1}, \quad X_{2} \rightarrow \omega^{k} X_{2}
$$

The former has $(n-1)^{2}$ elements while the latter has the $n-1$ elements. We confine ourselves to the case where we deform the theory by the lowest charge $x y$.

$t t^{*}$ equations: Here we briefly summarize the content of $t t^{*}$ equation following [14]. In an $N=2$ SUSY theory, there are two supersymmetry charges, $\bar{Q}$ and $Q$. Their property is

$$
(\bar{Q})^{2}=(Q)^{2}=0, \quad\{\bar{Q}, Q\}=H, \quad(\bar{Q})^{\dagger}=Q
$$

where $H$ is the hamiltonian. Topological theory is obtained by declaring $\bar{Q}$ to be a BRST operator and by identifying the BRST cohomology of $\bar{Q}$ with physical Hilbert space. The ground states $|i\rangle$ satisfy $\bar{Q}|i\rangle=0$, where we take the space to be a circle with periodic 
boundary condition. The topological operators $\phi_{i}$ are defined to be operators which commute with $\bar{Q}$, i.e. $\left[\bar{Q}, \phi_{i}\right]=0$, and are called chiral fields. These chiral fields form a ring because of OPE of two of them is $\bar{Q}$-closed.

$$
\phi_{i} \phi_{j}=C_{i j}^{k} \phi_{k}+[\bar{Q}, \Lambda]
$$

The CPT conjugate operators $\bar{\phi}_{i}$ commute with $Q$ and are called anti-chiral. There is an one-to-one correspondence between the ground states and the chiral operators: $\phi_{i}|0\rangle=$ $|i\rangle+\bar{Q}|\chi\rangle$. By definitions, the resulting state is a topological state. By acting the antichiral operators we get anti-topological state. The topological metric is defined by the two point function of chiral operators: $\eta_{i j}=\langle j \mid i\rangle$, and the ground state metric is defined as an inner product of a topological and an anti-topological state: $\langle\bar{j} \mid i\rangle=g_{i} \bar{j}$. They satisfy the reality condition:

$$
\eta^{-1} g\left(\eta^{-1}\right)^{*}=I
$$

The two point function $\eta_{i j}$ can be calculated by the Grothendieck residue

$$
\begin{aligned}
& \eta_{i j}=\left\langle\phi_{i} \phi_{j}\right\rangle=\operatorname{Res}\left[\phi_{i} \phi_{j}\right], \\
& \operatorname{Res}[\phi]=\frac{1}{(2 \pi i)^{n}} \int_{\Gamma} \frac{\phi(X) d X^{1} \wedge \cdots \wedge d X^{n}}{\partial_{1} W \partial_{2} W \cdots \partial_{n} W}
\end{aligned}
$$

where $W$ is the superpotential.

Now let us consider the perturbation of the action

$$
S=\int d^{2} z d^{4} \theta K+\left(\int d^{2} z d^{2} \theta W+c . c .\right)
$$

by the chiral operators with

$$
\delta S=\delta t_{i} \int d^{2} z d^{2} \theta \phi_{i}+c . c .
$$

where $t_{i}$ correspond to the (complex) couplings in the theory. As $t_{i}$ change the Ramond vacua change. The method of the computation of the metric $g_{i \bar{j}}$ is to study its behavior under perturbatuions which preserves the $N=2$ SUSY.

Analogous to Berry connection let us introduce the connections $A_{i}$ on the coupling constant space (holomorphic parameter space) given by

$$
A_{i a \bar{b}}=\left\langle\bar{b}\left|\partial_{i}\right| a\right\rangle .
$$

This connection measures the way the ground state subsector varies in the Hilbert space as the couplings change. Under a coupling constant dependent change of basis for the vacua, $A_{i}$ transforms as a gauge field. 
If we define the covariant derivative by $D_{i}=\partial_{i}-A_{i}$, then the metric $g_{i \bar{j}}$ is covariantly constant $D_{i} g_{i \bar{j}}=0=\bar{D}_{i} g_{i \bar{j}}$. One can prove the following equations [14]

$$
\begin{aligned}
& {\left[D_{i}, D_{j}\right]=\left[\bar{D}_{i}, \bar{D}_{j}\right]=0,} \\
& {\left[D_{i}, \bar{D}_{j}\right]=-\left[C_{i}, \bar{C}_{j}\right],}
\end{aligned}
$$

which are called $t t^{*}$ equations. If we choose holomorphic gauge $A_{\bar{i}}=0$ the second equation can be written as

$$
\bar{\partial}_{i}\left(g \partial_{j} g^{-1}\right)-\left[C_{j}, g\left(C_{i}\right)^{\dagger} g^{-1}\right]=0,
$$

which will be our main concern here.

\section{3. $t t^{*}$ equations for $\mathbb{C}^{1} / \mathbb{Z}_{n}$}

The $t t^{*}$ equations for the one field LG model under the deformation of most relevant operators $\left(x, x^{2}\right)$ were calculated by Cecotti and Vafa[14] and the results were various Toda systems. One can adopt their result to discuss the localized tachyon condensation in $\mathbb{C}^{1} / \mathbb{Z}_{n}$. The only thing one has to be careful is the renaming $n+1 \rightarrow n$ and the shift in power in the chiral ring elements associated with shift $\partial_{x} \rightarrow x \partial_{x}$. As a consequence, the evenness and oddness of various quantities are all reversed. So it is useful to rewrite the $t t^{*}$ equations in the present context and present notations.

\subsection{Deformation by $x$ : condensation of the most tachyonic state}

First we discuss the tachyon condensation by the most tachyonic state which has mass $m^{2}=2 \alpha^{\prime}(1 / n-1)$. The monomial representation of the corresponding operator is simply given by $x$ whose NS-charge is $1 / n$. So the tachyon condensation in the LG description is described by the superpotenial

$$
W=\frac{x^{n}}{n}-t x .
$$

Since the basic variable is $\log x$, the chiral ring is

$$
\mathcal{R}=\mathbb{C}[x] / \partial_{\log x} W=\left\{x, x^{2}, \cdots, x^{n-1}\right\} .
$$

The non-vanishing components of the topological metric $\eta$ are

$$
\eta_{i, n-i}=1, \text { for } i=1,2, \cdots, n-1 \text {. }
$$

Let $a_{i}:=g_{i \bar{i}}=\langle\bar{i} \mid i\rangle$. The reality condition gives

$$
\begin{aligned}
a_{i} a_{n-i} & =1, \\
\text { if } n=2 m+1, a_{m} & =1
\end{aligned}
$$


The non-vanishing components of structure constant matrix $C_{t}$ are

$$
\left(C_{t}\right)_{1}^{2}=\left(C_{t}\right)_{2}^{3}=\cdots=\left(C_{t}\right)_{n-2}^{n-1}=1,\left(C_{t}\right)_{n-1}^{1}=t .
$$

With these, the $t t^{*}$ equations can be calculated. ${ }^{1}$

$$
\begin{aligned}
& -\partial_{\bar{t}} \partial_{t} \log a_{1}=\frac{a_{2}}{a_{1}}-|t|^{2} \frac{a_{1}}{a_{n-1}} \\
& -\partial_{\bar{t}} \partial_{t} \log a_{i}=\frac{a_{i+1}}{a_{i}}-\frac{a_{i}}{a_{i-1}}, \quad i=2, \cdots, n-2 \\
& -\partial_{\bar{t}} \partial_{t} \log a_{n-1}=|t|^{2} \frac{a_{1}}{a_{n-1}}-\frac{a_{n-1}}{a_{n-2}} .
\end{aligned}
$$

By introducing new variables

$$
\begin{aligned}
q_{i} & =\log a_{i}-\frac{2 i-n}{2(n-1)} \log |t|^{2}, \\
z & =\frac{n-1}{n} t^{n /(n-1)},
\end{aligned}
$$

and $q_{n}=q_{1}, q_{0}=q_{n-1}$, the equations given in eqs.(3.6) take the form

$$
\partial_{z} \partial_{\bar{z}} q_{i}+e^{q_{i+1}-q_{i}}-e^{q_{i}-q_{i-1}}=0, \text { for } i=1, \cdots, n-1 .
$$

which is the $A_{n-2}$ Toda equations. By imposing the reality condition $q_{i}+q_{n-i}=0$, we can reduce the system to $C_{m}$ (if $n=2 m+1$ ) or $B C_{m}$ (if $n=2 m+2$ ) Toda system.

\subsection{Deformation by $x^{2}$}

$$
W=\frac{x^{n}}{n}-\frac{t}{2} x^{2}
$$

The chiral ring is the same as before. The topological metric $\eta$ are

$$
\eta_{i, j}=\delta_{i+j, n}+t \delta_{i, n-1} \delta_{j, n-1}, \text { for } i, j=1,2, \cdots, n-1 .
$$

The non-vanishing components of the ground state metric $g_{i j}$ is

$$
g_{i, \bar{i}}=a_{i}, \quad g_{n-1, \overline{1}}=b, \quad g_{1, \overline{n-1}}=\bar{b}
$$

The reality condition gives

$$
\begin{aligned}
a_{i} a_{n-i} & =1, \text { for } i=2, \cdots, n-2 \\
a_{n-1} & =1 / a_{1}+\frac{|t|^{2}}{4} a_{1}, \\
b & =\frac{1}{2} t a_{1}, \\
\text { and } \quad a_{m} & =1 \text { if } n=2 m+1,
\end{aligned}
$$

\footnotetext{
${ }^{1}$ For $\mathbb{C}^{1} / \mathbb{Z}_{n}$ problem, some of the $t t^{*}$ equations found by Cecotti and Vafa in [14] can be adopted for our purpose, while for $\mathbb{C}^{2} / \mathbb{Z}_{n}$ we really have to workout the corresponding systems.
} 
The non-vanishing components of structure constant matrix $C_{t}$ are

$$
\left(C_{t}\right)_{1}^{3}=\left(C_{t}\right)_{2}^{4}=\cdots=\left(C_{t}\right)_{n-3}^{n-1}=1,\left(C_{t}\right)_{n-2}^{2}=\left(C_{t}\right)_{n-1}^{3}=t
$$

According to whether $n$ is even or odd, the $t t^{*}$ equations are of different type.

For $n=2 m+1$, we define

$$
\begin{aligned}
q_{i} & =-\log a_{2 i-1} \quad \text { for } i=1,2, \cdots,[(m+1) / 2], \\
& =\log a_{2(m-i)+2} \quad \text { for } i=[(m+1) / 2]+1, \cdots, m
\end{aligned}
$$

Then the equations are

$$
\begin{aligned}
\partial \bar{\partial} q_{1} & =e^{q_{1}-q_{2}}-\frac{1}{4}|t|^{2} e^{-\left(q_{1}+q_{2}\right)} \\
\partial \bar{\partial} q_{2} & =e^{q_{2}-q_{3}}-e^{q_{1}-q_{2}}-\frac{1}{4}|t|^{2} e^{-\left(q_{1}+q_{2}\right)} \\
\partial \bar{\partial} q_{i} & =e^{q_{i}-q_{i+1}}-e^{q_{i-1}-q_{i}} \\
\partial \bar{\partial} q_{m} & =|t|^{2} e^{2 q_{m}}-e^{q_{m-1}-q_{m}}
\end{aligned}
$$

It is easy to re-scale the variables to make the equation as a standard $\tilde{B}_{m}:=D^{T}(S O(2 m+$ 1)) Toda form.

For even $n$, the $t t^{*}$ equations decouples into two independent Toda systems and we have two cases according to whether $n / 2$ is even or odd.

1. $n=4 m: a_{1}, a_{3}, \cdots, a_{2 m-1}$ satisfies $\tilde{B}_{m}$ Toda equations, while $a_{2}, a_{4}, \cdots, a_{2 m-2}$ satisfies $B C_{m-1}$ Toda.

2. $n=4 m+2: a_{1}, a_{3}, \cdots, a_{2 m-1}$ satisfies $B_{m}$ Toda equations, while $a_{2}, a_{4}, \cdots, a_{2 m}$ satisfies $C_{m}$ Toda.

\section{4. $t t^{*}$ equations for $\mathbb{C}^{2} / \mathbb{Z}_{n}$}

Now we consider $\mathbb{C}^{2} / \mathbb{Z}_{n(1,1)}$ with the generator $x y$ and we consider the condensation of most tachyonic operator, namely the generator $x y$ itself. Then the superpotential is given by

$$
W=\frac{x^{n}}{n}+\frac{y^{n}}{n}-t x y
$$

Since the $t t^{*}$ equations for this case has never been calculated, we do it here. Before we consider the mirror of $\mathbb{C}^{2} / \mathbb{Z}_{n}$, namely the orbifolded LG model, we first consider the generality of LG model itself without the orbifold action. 


\subsection{General aspects of LG model without orbifold action}

To see what is the non-vanishing elements of the ground state metric $g_{i \bar{j}}$ and the topological metric $\eta_{i j}$, we first consider the discrete symmetries of the superpotential.

Under the transformation

$$
x \rightarrow \omega^{a} x \text { and } y \rightarrow \omega^{b} y
$$

the superpotential $W$ is symmetric upto an over all phase $\omega^{*}$ which is cancelled by transforming the $\theta \rightarrow \omega^{(a+b) / 2} \theta$. By requiring invariance of the action we have

$$
\omega^{a n}=\omega^{b n}=\omega^{a+b} .
$$

Without loss of generality, we can set:

$$
a=1, \quad b=n-1, \quad \omega^{n(n-2)}=1,
$$

up to an equivalence class. Now $\eta_{i j}$ is given by the Griffith residue

$$
\eta_{i j}=\left\langle x^{i_{1}} y^{j_{1}} \cdot x^{i_{2}} y^{j_{2}}\right\rangle=\int \frac{d x}{x} \wedge \frac{d y}{y} \frac{x^{i_{1}+i_{2}} y^{j_{1}+j_{2}}}{\left(x^{n}-t x y\right)\left(y^{n}-t x y\right)} .
$$

The non-vanishing components should be invariant under the above symmetry transformations. This requires

$$
\left(i_{1}+i_{2}-2\right)+(n-1)\left(j_{1}+j_{2}-2\right) \equiv 0 \bmod n(n-2) .
$$

The selection rule is given by the solutions to this equation:

$$
i_{1}+i_{2}=j_{1}+j_{2}=I \equiv 2 \bmod (n-2) .
$$

For the actual value, we perform explicit residue calculations, whose example is given in the appendix. We found that

- $I=2$ case: We have one solution $i_{1}=i_{2}=1, j_{1}=j_{2}=1$. However, it turns out that $\eta=0$ in this case.

- $I=n$ case: $i_{1}=j_{1}=i, i_{2}=j_{2}=n-1-i, i=1, \cdots, n-1$. These are $n-1$ cross diagonal elements. All of them are 1.

- $I=2(n-1)$ case: There is only one element $i_{1}=j_{1}=i_{2}=j_{2}=n-1$, and the value is $\eta_{i j}=t^{2}$. 
One can find similar selection rule for the metric element $g_{i \bar{j}}=\left\langle x^{i_{1}} y^{j_{1}} x^{\bar{i}_{2}} y^{\bar{j}_{2}}\right\rangle$. For non-vanishing elements we need

$$
\omega^{i_{1}-i_{2}+(n-1) j_{1}-(n-1) j_{2}}=1 \text { with } \omega^{n(n-2)}=1,
$$

which gives

$$
i_{1}-i_{2}+(n-1)\left(j_{1}-j_{2}\right) \equiv 0 \bmod n(n-2) .
$$

As consequences we have

$$
i_{1}-i_{2}=j_{1}-j_{2} \equiv 0 \bmod (n-2)
$$

There are two cases:

- $i_{1}=i_{2}, j_{1}=j_{2}$. These are the diagonal elements of $g_{i \bar{j}}$. We define $a_{i j}:=\left\langle\bar{x}^{i} \bar{y}^{j} \mid x^{i} y^{j}\right\rangle$.

- $j_{1}-j_{2}=i_{1}-i_{2}= \pm(n-2)$, namely

$$
\begin{aligned}
i_{1}=j_{1}=n-1, & i_{2}=j_{2}=1, \text { or } \\
i_{1}=j_{1}=1, & i_{2}=j_{2}=n-1,
\end{aligned}
$$

which are the two corner-most off-diagonal elements. we call it as $\bar{b}$ and $b$ respectively. The calculation of $t t^{*}$ equation for general LG model is cumbersome. We now simplify our life by performing the orbifold action. The simplification is given by reducing the number of chiral ring elements from $(n-1)^{2}$ to $n-1$.

\subsection{Orbifolded LG: the mirror of $\mathbb{C}^{2} / \mathbb{Z}_{n}$}

The chiral ring in this case is generated by $x y$ :

$$
\mathcal{R}=\left\{x y,(x y)^{2}, \cdots,(x y)^{n-1}\right\} .
$$

Let

$$
a_{i}=\langle\bar{x} \bar{y})^{i}\left|(x y)^{i}\right\rangle=e^{-q_{i}} .
$$

The topological metric can be calculated to give

$$
\eta=\eta_{i j}=\delta_{i+j, n}+t^{2} \delta_{i, n-1} \delta_{j, n-1} .
$$

The structure constants are

$$
\left(C_{t}\right)_{i}^{j}=\delta_{i, j-1}+t^{2} \delta_{i, n-1} \delta_{j, 2}
$$

The reality condition gives

$$
a_{i} a_{n-i}=1(\text { for } i=2, \cdots, n-1), \quad a_{n-1}=1 / a_{1}+|t|^{4} a_{1} / 4 .
$$


With all these, we can calculate the $t t^{*}$ equations. Let's introduce a parameter $s$ by

$$
n=2 m+2-s, \quad s=0,1
$$

Then $t t^{*}$ equation can be written as

$$
\begin{aligned}
-\partial_{\bar{t}} \partial_{t} \log a_{1} & =\frac{a_{2}}{a_{1}}-\frac{1}{4}|t|^{4} a_{1} a_{2} \\
-\partial_{\bar{t}} \partial_{t} \log a_{2} & =\frac{a_{3}}{a_{2}}-\frac{a_{2}}{a_{1}}-\frac{1}{4}|t|^{4} a_{1} a_{2} \\
-\partial_{\bar{t}} \partial_{t} \log a_{i} & =\frac{a_{i+1}}{a_{i}}-\frac{a_{i}}{a_{i-1}}, \quad i=3, \cdots, m-1 \\
-\partial_{\bar{t}} \partial_{t} \log a_{m} & =a_{m}^{-(1+s)}-\frac{a_{m}}{a_{m-1}}
\end{aligned}
$$

Let's re-scale the variables to eliminate $|t|$ 's and other coefficients.

$$
a_{i}=\delta_{i}|t|^{\alpha_{i}} e^{-q_{i}}, \quad \zeta=\gamma t^{\beta}
$$

Then

$$
\begin{aligned}
& \alpha_{1}=-2, \quad \alpha_{2}=2 \beta-4 \\
& \alpha_{m}=(m-1)(2 \beta-2)+\alpha_{1}=-\frac{2(\beta-1)}{1+s}, \\
& \beta=1+\frac{1+s}{1+(1+s)(m-1)} \\
& \alpha_{k}=-2 \frac{(1+s)(m-k)+1}{1+(1+s)(m-1)} \\
& \delta_{1}=2, \quad \delta_{m}=\delta_{1}(\gamma \beta)^{2(m-1)} \\
& \gamma=\beta^{-1}\left(\frac{1}{2}\right)^{\frac{1+s}{1+2(m-1)(1+s)}}
\end{aligned}
$$

Then

$$
\begin{aligned}
\partial \bar{\partial} q_{1} & =e^{q_{1}-q_{2}}-e^{-\left(q_{1}+q_{2}\right)}, \\
\partial \bar{\partial} q_{2} & =e^{q_{2}-q_{3}}-e^{q_{1}-q_{2}}-e^{-\left(q_{1}+q_{2}\right)}, \\
\partial \bar{\partial} q_{i} & =e^{q_{i}-q_{i+1}}-e^{-\left(q_{i-1}-q_{i}\right)}, i=3, \cdots, m-1 \\
\partial \bar{\partial} q_{m} & =e^{(1+s) q_{m}}-e^{\left(q_{m-1}-q_{m}\right)}
\end{aligned}
$$

One can bring this equation to the Toda equations: for $s=0$ (even $n$ ) these are $B_{m}$ Toda and for $s=1(\operatorname{odd} n)$ they are $\tilde{B}_{m}=D^{T}(S O(2 m+1))$ Toda system. It is a curious fact that the Toda system appearing in the deformation of $\mathbb{C}^{2} / Z_{n}$ by $x y$ is identical to that appearing in the deformation of $D_{n}$ singularity $\left(W=\frac{1}{n-1} x^{n-1}+x y^{2}\right)$ by $x$. Notice that the mirror geometric correspondence of $D_{n}$ singularity has not been clear at all and is still not clear. 
The charge matrix $Q=g \partial_{\tau} g^{-1}-1$ with $\tau=\log \lambda$ can be calculated to be given by

$$
Q=\left(\begin{array}{ccccc}
\frac{2-n}{n}+a_{11} \partial_{\tau} a_{11}^{-1} & 0 & \cdots & 0 & 0 \\
0 & \frac{4-n}{n}+a_{22} \partial_{\tau} a_{22}^{-1} & \cdots & 0 & 0 \\
\ldots & \cdots & \cdots & \cdots & \cdots \\
0 & 0 & \cdots \frac{n-4}{n}-a_{22} \partial_{\tau} a_{22}^{-1} & 0 \\
t^{2} a_{11} \partial_{\tau} a_{11}^{-1} & 0 & \cdots & 0 & \frac{n-2}{n}-a_{11} \partial_{\tau} a_{11}^{-1}
\end{array}\right)
$$

Notice that we do not need to perform the diagonalization to get the eigenvalues of $Q$. In terms of $q_{i j}$ and $\lambda(=\zeta)$, and if we look at the $|\lambda|=z$ dependence only, the tachyon potential can be identified as

$$
V=2 Q_{\max }=-z \partial_{z} q_{11}(z)
$$

So far, no mathematical literature on the solution to the equation is available. However, from the physical intuition that in the final stage of tachyon condensation there is no nontrivial chiral primaries with charge other than 0 and also from the experience from the low $n$ cases, we expect that the potential monotonically decrease from the value $2 / n-1$ at $t=0$ to 0 at $t \rightarrow \infty$.

\section{Discussion}

In this paper, we worked out equations describing the tachyon condensation in orbifolds $\mathbb{C}^{1} / \mathbb{Z}_{n}$ and $\mathbb{C}^{2} / \mathbb{Z}_{n}$ and the tachyon potential. The resulting equations can be identified as various Toda systems when we consider the condensation by the lowest tachyon. We find that the potential is calculated as a solution to Toda equations and conjectured to be monotonically decreasing.

It is interesting to notice that the Toda system appearing as $t t^{*}$ equation in the deformation of $\mathbb{C}^{2} / \mathbb{Z}_{n}$ by $x y$ is identical to that of $D_{n}$ singularity by $x$. It is not clear whether this signalize the possibility to identify the geometry corresponding to $D_{n}$ singularity as $\mathbb{C}^{2} / \mathbb{Z}_{n}$. It is tantalizing problem to answer to the question what is the geometry corresponding to the general singularities along the line of the idea that $A_{N-1}$ singularity corresponds to the $\mathbb{C} / \mathbb{Z}_{N}$.

We should point out that we considered the string theories without GSO projection only when we discuss the $x y$ deformation of $\mathbb{C}^{2} / \mathbb{Z}_{n}$ and $x^{2}$ deformation of $\mathbb{C}^{1} / \mathbb{Z}_{n}$. The reason is simply that if we impose the type II projection rule, the above operators are projected out and remaining spectrum gives us complicated reality conditions so that the resulting $t t^{*}$ equations are technically beyond our reach so far. Related problem arises when the daughter theory is also an orbifold $\mathbb{C}^{1} / \mathbb{Z}_{n}$ background. 
One of the prime difficulty in the solution comes from the constraints coming from the reality condition. For the lowest deformations of $\mathbb{C} / Z_{n}$ and $\mathbb{C}^{2} / \mathbb{Z}_{n}$, the reality conditions can be easily solved as we have seen in the main sections. However, for $\mathbb{C}^{3} / \mathbb{Z}_{n}$ with lowest perturbation $x y z$ or for $\mathbb{C} / \mathbb{Z}_{n}$ case with non-trivial deformation like $x^{3}$, the solutions are non-trivial. If we adopt an special ansatz, it the resulting solutions corresponds to the wrong boundary conditions as we described at the end of the appendices.

Of course most immediate question is whether we can write down analytic solution of the toda systems we found with appropriate boundary conditions. So far no mathematical literature is available to our knowledge. We wish to report on these issues in later publications.

\section{A. $\mathbb{C}^{3} / \mathbb{Z}_{n}$}

In this appendix we present a somewhat incomplete result on $\mathbb{C}^{3} / \mathbb{Z}_{n}$. We spent long time without success in getting a simple system with solutions satisfying proper boundary conditions. We point out what are the difficulty through an example $n=5$ case. We also give a comparison with similar system $\mathbb{C} / \mathbb{Z}_{5} \rightarrow \mathbb{C} / \mathbb{Z}_{3}$.

The superpotential is

$$
W=x^{n} / n+y^{n} / n+z^{n} / n-t x y z .
$$

We work in the basis

$$
\left\{x y z,(x y z)^{2}, \cdots,(x y z)^{n-1}\right\} .
$$

Under the transformation

$$
x \rightarrow \omega^{a} x, \quad y \rightarrow \omega^{b} y, \quad z \rightarrow \omega^{c} z,
$$

with well chosen $a, b$, and $c$, the superpotential becomes invariant. The choice is

$$
b=a, \quad c=a(n-2), \quad \text { with } \omega^{a n(n-3)}=1 .
$$

From this we can get the non-vanishing components of the topological metric $\eta_{i j}$ and metric $g_{j i}$. First for $\eta_{i j}$,

$$
\eta=\int \frac{(x y z)^{I} d x \wedge d y \wedge d z}{\left(x^{n-1}-t y z\right)\left(y^{n-1}-t x z\right)\left(z^{n-1}-t x y\right)} .
$$

In order for this to be invariant under eq. A.4,

$$
\operatorname{an}(I-1)=0 \bmod a n(n-3)
$$


which means some components of $\eta$ are non-zero. One can find and calculate the nonvanishing values of $\eta$ :

$$
0: \text { when } I=1 \text { and } n-1:, \quad t^{3}: \text { when } I=2 n-3 \text {. }
$$

For the metric

$$
g_{\bar{j} i}=\left\langle(x y z)^{\bar{j}} \mid(x y z)^{i}\right\rangle
$$

to be invaraint we obtain

$$
a n(i-j)=0, \bmod a n(n-3) .
$$

Therefore we obtain the diagonal of the metric (we spell out them as $a_{i i}$ ) and the offdiagonal components (denoted by $b_{1}$ and $b_{2}$ and their conjugates).

One can find $C_{t}$ :

$$
\left(C_{t}\right)_{1}^{2}=\left(C_{t}\right)_{2}^{3}=\cdots=\left(C_{t}\right)_{n-3}^{n-2}=1, \quad\left(C_{t}\right)_{3}^{n-1}=t^{3} .
$$

The difficult part is the reality condition. Although we can solve it with the ansatz

$$
b_{1}=a_{11} t^{3} / 2, \quad b_{2}=a_{22} t^{3} / 2,
$$

there is no guarantee that its consequence is consistent with the boundary conditions we have to impose. With all these we can write down the $t t^{*}$ equation, but for $n \geq 6$ we get inconsistent equation like

$$
a_{11}=0(n=6) \quad \text { or } \quad a_{11} a_{33}=0(n>6)
$$

which violates regularity of the metric. The $t t^{*}$ equation for general $\mathbb{C}^{3} / \mathbb{Z}_{n}$ is not simple. Main reason is the absence of the simple solution to the reality constraint. We give some detail for $n=5$.

\section{A.1 $t t^{*}$ euqation for $\mathbb{C}^{3} / \mathbb{Z}_{5} \rightarrow \mathbb{C}^{3} / \mathbb{Z}_{3}$}

In this case the superpotential is written as

$$
W=x^{5} / 5+y^{5} / 5+z^{5} / 5-t x y z .
$$

The symmetry that the superpotential has is

$$
x \rightarrow \omega^{a} x \quad y \rightarrow \omega^{a} y \quad z \rightarrow \omega^{3 a} z \text { with } \omega^{10 a}=1 .
$$

This restricts the topological metric and metric to be

$$
\eta_{i j}=\left(\begin{array}{cccc}
0 & 0 & 0 & 1 \\
0 & 0 & 1 & 0 \\
0 & 1 & 0 & t^{3} \\
1 & 0 & t^{3} & 0
\end{array}\right), \quad g_{\bar{j} i}=\left(\begin{array}{cccc}
a_{11} & 0 & \bar{b}_{1} & 0 \\
0 & a_{22} & 0 & \bar{b}_{2} \\
b_{1} & 0 & a_{33} & 0 \\
0 & b_{2} & 0 & a_{44}
\end{array}\right),
$$


and the multiplication matrix for the perturbing chiral ring element $x y z$ is

$$
\left(C_{t}\right)_{i}^{j}=\left(\begin{array}{cccc}
0 & 1 & 0 & 0 \\
0 & 0 & 1 & 0 \\
0 & 0 & 0 & 1 \\
0 & 0 & t^{3} & 0
\end{array}\right) .
$$

We take the basis as

$$
\left\{(x y z),(x y z)^{2},(x y z)^{3},(x y z)^{4}\right\} .
$$

We list here all $t t^{*}$ equations for $\mathbb{C}^{3} / \mathbb{Z}_{5}$ case.

$$
\begin{aligned}
& \partial_{\bar{t}}\left(\frac{a_{3} \partial_{t} a_{1}-b_{1} \partial_{t} \bar{b}_{1}}{-a_{1} a_{3}+b_{1} \bar{b}_{1}}\right)=\frac{a_{2} a_{3}-b_{1} \bar{b}_{2}}{a_{1} a_{3}-b_{1} \bar{b}_{1}}, \quad \partial_{\bar{t}}\left(\frac{b_{1} \partial_{t} a_{3}-a_{3} \partial_{t} b_{1}}{a_{1} a_{3}-b_{1} \bar{b}_{1}}\right)=\frac{a_{4} b_{1}-a_{3} b_{2}}{-a_{1} a_{3}+b_{1} \bar{b}_{1}} \\
& \partial_{\bar{t}}\left(\frac{a_{4} \partial_{t} a_{2}-b_{2} \partial_{t} \bar{b}_{2}}{-a_{2} a_{4}+b_{2} \bar{b}_{2}}\right)= \\
& \frac{-a_{2}^{2} a_{3} a_{4}+a_{1} a_{3}^{2}\left(a_{4}-\bar{t}^{3} b_{2}\right)+a_{2}\left(a_{4} b_{1}+a_{3} b_{2}\right) \bar{b}_{2}-b_{1}\left(a_{3} \bar{b}_{1}\left(a_{4}-\bar{t}^{3} b_{2}\right)+b_{2} \bar{b}_{2}^{2}\right)}{\left(a_{1} a_{3}-b_{1} \bar{b}_{1}\right)\left(a_{2} a_{4}-b_{2} \bar{b}_{2}\right)}, \\
& \partial_{\bar{t}}\left(\frac{b_{2} \partial_{t} a_{4}-a_{4} \partial_{t} b_{2}}{a_{2} a_{4}-b_{2} \bar{b}_{2}}\right)= \\
& \frac{-t^{3} a_{3} a_{4} b_{1} \bar{b}_{1}+|t|^{6} a_{3} b_{1} \bar{b}_{1} b_{2}+t^{3} a_{1} a_{3}^{2}\left(a_{4}-\bar{t}^{2} b_{2}\right)+a_{2} a_{4}\left(a_{4} b_{1}-a_{3} b_{2}\right)-a_{4} b_{1} b_{2} \bar{b}_{2}+a_{3} b_{2}^{2} \bar{b}_{2}}{\left(a_{1} a_{3}-b_{1} \bar{b}_{1}\right)\left(a_{2} a_{4}-b_{2} \bar{b}_{2}\right)}, \\
& \partial_{\bar{t}}\left(\frac{\bar{b}_{1} \partial_{t} a_{1}-a_{1} \partial_{t} \bar{b}_{1}}{a_{1} a_{3}-b_{1} \bar{b}_{1}}\right)= \\
& \frac{a_{1}\left(-b_{2} \bar{b}_{2}^{2}+a_{3} \bar{b}_{1}\left(-a_{4}+\bar{t}^{3} \bar{b}_{2}\right)+a_{2}\left(-|t|^{6} a_{3} \bar{b}_{1}+a_{4} \bar{b}_{2}\right)\right.}{\left(a_{1} a_{3}-b_{1} \bar{b}_{1}\right)\left(a_{2} a_{4}-b_{2} \bar{b}_{2}\right)} \\
& +\frac{\bar{b}_{1}\left(-a_{2}^{2} a_{4}+b_{1} \bar{b}_{1}\left(a_{4}-\bar{t}^{3} b_{2}-t^{3} b_{2}\right)+a_{2}\left(|t|^{6} b_{1} \bar{b}_{1}+b_{2} \bar{b}_{2}\right)\right)}{\left(a_{1} a_{3}-b_{1} \bar{b}_{1}\right)\left(a_{2} a_{4}-b_{2} \bar{b}_{2}\right)}, \\
& \partial_{\bar{t}}\left(\frac{a_{1} \partial_{t} a_{3}-\bar{b}_{1} \partial_{t} b_{1}}{-a_{1} a_{3}+b_{1} \bar{b}_{1}}\right)= \\
& \frac{\bar{b}_{1}\left(a_{2}\left(|t|^{6} a_{3} b_{1}-a_{4} b_{2}\right)+b_{2}^{2} \bar{b}_{2}+a_{3} b_{1}\left(a_{4}-\bar{t}^{3} b_{2}-t^{3} \bar{b}_{2}\right)\right)}{\left(a_{1} a_{3}-b_{1} \bar{b}_{1}\right)\left(a_{2} a_{4}-b_{2} \bar{b}_{2}\right)} \\
& +\frac{a_{1}\left(a_{2}\left(-|t|^{6} a_{3}^{2}+a_{4}^{2}\right)-a_{4} b_{2} \bar{b}_{2}+a_{3}^{2}\left(-a_{4}+\bar{t}^{3} b_{2}+t^{3} \bar{b}_{2}\right)\right)}{\left(a_{1} a_{3}-b_{1} \bar{b}_{1}\right)\left(a_{2} a_{4}-b_{2} \bar{b}_{2}\right)}, \\
& \partial_{\bar{t}}\left(\frac{\bar{b}_{2} \partial_{t} a_{2}-a_{2} \partial_{t} \bar{b}_{2}}{-a_{2} a_{4}+b_{2} \bar{b}_{2}}\right)= \\
& \frac{a_{1}\left(a_{2}\left(\bar{t}^{3} a_{3}^{2}-a_{4} \bar{b}_{2}\right)+\bar{b}_{2}\left(-a_{3}^{2}+b_{2} \bar{b}_{2}\right)\right)+\bar{b}_{1}\left(a_{2}^{2} a_{4}+a_{3} b_{1} \bar{b}_{2}-a_{2}\left(\bar{t}^{3} a_{3} b_{1}+b_{2} \bar{b}_{2}\right)\right)}{\left(a_{1} a_{3}-b_{1} \bar{b}_{1}\right)\left(a_{2} a_{4}-b 2 \bar{b}_{2}\right)}, \\
& \partial_{\bar{t}}\left(\frac{a_{2} \partial_{t} a_{4}-\bar{b}_{2} \partial_{t} b_{2}}{-a_{2} a_{4}+b_{2} \bar{b}_{2}}\right)= \\
& \frac{a_{1}\left(a_{2}\left(|t|^{6} a_{3}^{2}-a_{4}^{2}\right)-\left(t^{3} a_{3}^{2}+a_{4} b_{2}\right) \bar{b}_{2}+\bar{b}_{1}\left(a_{2}\left(-|t|^{6} a_{3} b_{1}+a_{4} b_{2}\right)+\left(t^{3} a_{3} b_{1}-b_{2}^{2}\right) \bar{b}_{2}\right)\right.}{\left(a_{1} a_{3}-b_{1} \bar{b}_{1}\right)\left(a_{2} a_{4}-b_{2} \bar{b}_{2}\right)} .
\end{aligned}
$$


These equations should be supplemented by the constraints coming from the reality condition:

$$
\begin{aligned}
\left(\vec{t}^{2} a_{1}+\bar{b}_{1}\right)\left(-t^{3} a_{2}+b_{2}\right)+a_{1}\left(a_{4}-t^{3} \bar{b}_{2}\right) & =1 \\
a_{2}\left(a_{3}-t^{3} \bar{b}_{1}\right)+\left(-t^{3} a_{1}+b_{1}\right)\left(-\bar{t}^{3} a_{2}+\bar{b}_{2}\right) & =1 \\
a_{2}\left(a_{3}-\bar{t}^{3} b_{1}\right)+b_{1} \bar{b}_{2} & =1, \\
\bar{b}_{1} b_{2}+a_{1}\left(a_{4}-\bar{t}^{3} b_{2}\right) & =1, \\
a_{2} \bar{b}_{1}+a_{1}\left(-\bar{t}^{3} a_{2}+b_{2}\right) & =0 \\
\left(a_{3}-\bar{t}^{3} b_{1}\right)\left(-t^{3} a_{2}+b_{2}\right)+b_{1}\left(a_{4}-t^{3} \bar{b}_{2}\right) & =0 \\
\left(a_{3}-t^{3} \bar{b}_{1}\right) b_{2}+\left(-t^{3} a_{1}+b_{1}\right)\left(a_{4}-\bar{t}^{3} b_{2}\right) & =0 .
\end{aligned}
$$

We may wonder whether we can find some ansatz to make the $t t^{*}$ equations simple. We will try an obvious one and show how it does not work. Let's try an ansatz given by

$$
b_{1}=a_{1} t^{3} / 2, \quad b_{2}=a_{2} t^{3} / 2,
$$

Together with the reality condition above ansatz gives

$$
a_{3}=1 / a_{2}+|t|^{6} a_{1} / 4, \quad a_{4}=1 / a_{1}+|t|^{6} a_{2} / 4, \quad \text { with } a_{1} a_{4}=a_{2} a_{3} .
$$

From $t t^{*}$ equation we get the relation

$$
a_{2}=-4 /\left(|t|^{6} a_{1}\right)
$$

Then finally we get

$$
-\partial_{\bar{t}} \partial_{t} \log a_{1}=-4 /\left(|t|^{6} a_{1}^{2}\right)
$$

Now let us define

$$
\zeta=t^{-2} \text { and } a_{1}=e^{\phi},
$$

the eq. A.23) becomes Liouville equation

$$
\partial_{\bar{\zeta}} \partial_{\zeta} \phi=e^{-2 \phi}
$$

This has an analytic solution. However this turns out to have incorrect behavior.

Let us rescale

$$
x=\lambda^{1 / 5} \tilde{x}, \quad y=\lambda^{1 / 5} \tilde{y}, \quad z=\lambda^{1 / 5} \tilde{z} \quad \lambda=t^{2 / 5} .
$$

Then we have the super potential is rescaled to give

$$
\tilde{W}=\lambda\left(\tilde{x}^{5} / 5+\tilde{y}^{5} / 5+\tilde{z}^{5} / 5-\tilde{x} \tilde{y} \tilde{z}\right)
$$


The charge can be calculated by the relation

$$
Q=\tilde{g} \partial_{\tau} \tilde{g}^{-1}-3 / 2
$$

The correlation functions are rescaled to give

$$
a_{i}=\left\langle(\bar{x} \bar{y} \bar{z})^{i} \mid(x y z)^{i}\right\rangle=|\lambda|^{6 i / 5}\left\langle(\overline{\tilde{x}} \overline{\tilde{y}} \overline{\tilde{z}})^{i} \mid(\tilde{x} \tilde{y} \tilde{z})^{i}\right\rangle:=|\lambda|^{6 i / 5} b_{i}
$$

where $b_{i}$ are components of $\tilde{g}$. Therefore the charge is

$$
Q_{\min }=\frac{1}{2} b_{1}|\lambda| \partial_{|\lambda|} b_{1}^{-1}-\frac{3}{2}=-\frac{9}{10}-\frac{1}{2}|\lambda| \frac{d a_{1}}{d|\lambda|}=-\frac{9}{10}+\frac{5}{2}|\zeta| \frac{d a_{1}}{d|\zeta|},
$$

where we have used $\lambda=t^{2 / 5}$ and $\zeta=t^{-2}$. So, the required boundary conditions are

$$
|\zeta| \frac{d a_{1}}{d|\zeta|} \rightarrow 0 \text { if }|\zeta| \rightarrow \infty(U V), \quad|\zeta| \frac{d a_{1}}{d|\zeta|} \rightarrow \frac{18}{50} \text { if }|\zeta| \rightarrow 0(I R)
$$

The general solution for Liouville equation

$$
-\partial_{\zeta} \partial_{\bar{\zeta}} \phi+e^{-2 \phi}=\frac{1-a}{2} \delta^{(2)}(z)
$$

is 15

$$
e^{-2 \phi}=\frac{\partial A(\zeta) \partial B(\bar{\zeta})}{(1-A(\zeta) B(\bar{\zeta}))^{2}} .
$$

There are three types of solutions according to the region of $a$ : Elliptic ( $a$ real), Parabolic $(a \rightarrow 0)$, Hyperbolic ( $a$ pure imaginary). However, none of such solutions can match the boundary conditions. Therefore the ansatz given in eq.(A.20) does not work.

\section{A.2 $t t^{*}$ euqation for $\mathbb{C} / \mathbb{Z}_{5} \rightarrow \mathbb{C} / \mathbb{Z}_{3}$}

Similar problem arise when we study $\mathbb{C}^{1} / \mathbb{Z}_{5}$ with non-trivial tachyon perturbation, namely,

$$
W=x^{5} / 5-t x^{3} / 3
$$

Therefore the basis is

$$
\left\{x, x^{2}, x^{3}, x^{4}\right\}
$$

The symmetry restricts the topological metric and metric which is given by

$$
\eta=\left(\begin{array}{cccc}
0 & 0 & 0 & 1 \\
0 & 0 & 1 & 0 \\
0 & 1 & 0 & t \\
1 & 0 & t & 0
\end{array}\right), \quad g=\left(\begin{array}{cccc}
a_{1} & 0 & \bar{b}_{1} & 0 \\
0 & a_{2} & 0 & \bar{b}_{2} \\
b_{1} & 0 & a_{3} & 0 \\
0 & b_{2} & 0 & a_{4}
\end{array}\right)
$$


and $C_{t}$ is given by

$$
C_{t}=\left(\begin{array}{cccc}
0 & 0 & 0 & 1 \\
0 & 0 & t & 0 \\
0 & 0 & 0 & t \\
0 & 0 & t^{2} & 0
\end{array}\right)
$$

We take ansatz to solve reality condition

$$
b_{1}=a_{1} t / 2, \quad b_{2}=a_{2} t / 2
$$

and the result is

$$
a_{3}=1 / a_{2}+|t|^{2} a_{1} / 4, \quad a_{4}=1 / a_{1}+|t|^{2} a_{2} / 4, \quad \text { with } a_{1} a_{4}=a_{2} a_{3} .
$$

$t t^{*}$ equation gives one more restriction to $a_{2}$

$$
a_{2}=|t| a_{1}
$$

Then we finally get the simplified $t t^{*}$ equation

$$
-\partial_{\bar{t}} \partial_{t} \log a_{1}=1 / a_{1}^{2}-|t|^{6} a_{1}^{2} / 16
$$

If we define $a_{1}=1 / y$, then the equation becomes

$$
\partial_{\bar{t}} \partial_{t} \log y=y^{2}-|t|^{6} / 16 y^{2} \text {. }
$$

By introducing change of variables

$$
\zeta=(2 / 5) t^{5 / 2}, \quad y=\sqrt{1 / 2}|t|^{3 / 2} e^{u / 2},
$$

one can rewite this equation as sinh-Gordon

$$
\partial_{\bar{\zeta}} \partial_{\zeta} u=\sinh u
$$

The solution to this equation is describing the solution whose associated charges decrease to zero, which is not what we want here. Hence we see that the ansatz (A.38) above is not a proper one. Then the equation is given by $t t^{*}$ equation

$$
\begin{aligned}
\partial_{\bar{t}}\left(\frac{a_{3} \partial_{t} a_{1}-b_{1} \partial_{t} \bar{b}_{1}}{-a_{1} a_{3}+\left|b_{1}\right|^{2}}\right) & =\frac{a_{4}\left(a_{3}-\bar{t} b_{1}\right)}{a_{1} a_{3}-\left|b_{1}\right|^{2}}, \\
\partial_{\bar{t}}\left(\frac{b_{1} \partial_{t} a_{3}-a_{3} \partial_{t} b_{1}}{a_{1} a_{3}-\left|b_{1}\right|^{2}}\right) & =\frac{t a_{4}\left(a_{3}-\bar{t} b_{1}\right)}{a_{1} a_{3}-\left|b_{1}\right|^{2}}, \\
\partial_{\bar{t}}\left(\frac{a_{4} \partial_{t} a_{2}-b_{2} \partial_{t} \bar{b}_{2}}{-a_{2} a_{4}+\left|b_{2}\right|^{2}}\right) & =\frac{|t|^{2} a_{3}\left(a_{4}-\bar{t} b_{2}\right)}{a_{2} a_{4}-\left|b_{2}\right|^{2}}, \\
\partial_{\bar{t}}\left(\frac{b_{2} \partial_{t} a_{4}-a_{4} \partial_{t} b_{2}}{a_{2} a_{4}-\left|b_{2}\right|^{2}}\right) & =\frac{t|t|^{2} a_{3}\left(a_{4}-\bar{t} b_{2}\right)}{a_{2} a_{4}-\left|b_{2}\right|^{2}},
\end{aligned}
$$




$$
\begin{aligned}
& \partial_{\bar{t}}\left(\frac{\bar{b}_{1} \partial_{t} a_{1}-a_{1} \partial_{t} \bar{b}_{1}}{a_{1} a_{3}-\left|b_{1}\right|^{2}}\right)=\frac{\bar{t} a_{1}\left(a_{2}\left(a_{4}^{2}-|t|^{2} t a_{3} \bar{b}_{1}\right)-a_{4}\left|b_{2}\right|^{2}+t a_{3} \bar{b}_{1}\left(-a_{4}+\bar{t} b_{2}+t \bar{b}_{2}\right)\right)}{\left(a_{1} a_{3}-\left|b_{1}\right|^{2}\right)\left(a_{2} a_{4}-\left|b_{2}\right|^{2}\right)} \\
& +\frac{\bar{b}_{1}\left(a_{2}\left(-a_{4}^{2}+|t|^{4}\left|b_{1}\right|^{2}\right)-|t|^{2}\left|b_{1}\right|^{2}\left(\bar{t} b_{2}+t \bar{b}_{2}+a_{4}\left(|t|^{2}\left|b_{1}\right|^{2}+\left|b_{2}\right|^{2}\right)\right)\right.}{\left(a_{1} a_{3}-\left|b_{1}\right|^{2}\right)\left(a_{2} a_{4}-\left|b_{2}\right|^{2}\right)}, \\
& \partial_{\bar{t}}\left(\frac{a_{1} \partial_{t} a_{3}-\bar{b}_{1} \partial_{t} b_{1}}{-a_{1} a_{3}+\left|b_{1}\right|^{2}}\right)=\frac{t\left(\bar{t} a_{1}\left(a_{2}\left(-|t|^{2} a_{3}^{2}+a_{4}^{2}\right)-a_{4}\left|b_{2}\right|^{2}+a_{3}^{2}\left(-a_{4}+\bar{t} b_{2}+t \bar{b}_{2}\right)\right)\right)}{\left(a_{1} a_{3}-\left|b_{1}\right|^{2}\right)\left(a_{2} a_{4}-\left|b_{2}\right|^{2}\right)} \\
& +\frac{t\left(\bar{b}_{1}\left(a_{2}\left(-a_{4}^{2}+|t|^{2} \bar{t} a_{3} b_{1}\right)+a_{4}\left|b_{2}\right|^{2}-\bar{t} a_{3} b_{1}\left(-a_{4}+\bar{t} b_{2}+t \bar{b}_{2}\right)\right)\right)}{\left(a_{1} a_{3}-\left|b_{1}\right|^{2}\right)\left(a_{2} a_{4}-\left|b_{2}\right|^{2}\right)}, \\
& \partial_{\bar{t}}\left(\frac{\bar{b}_{2} \partial_{t} a_{2}-a_{2} \partial_{t} \bar{b}_{2}}{a_{2} a_{4}-\left|b_{2}\right|^{2}}\right)=\frac{a_{2}\left(a_{4}\left(\bar{t} b_{1}+t \bar{b}_{1}\right) \bar{b}_{2}-a_{3}\left(|t|^{2} \bar{t}\left|b_{1}\right|^{2}+a_{4} \bar{b}_{2}\right)\right)}{\left(a_{1} a_{3}-\left|b_{1}\right|^{2}\right)\left(a_{2} a_{4}-\left|b_{2}\right|^{2}\right)} \\
& +\frac{|t|^{2} a_{1}\left(a_{2}\left(\bar{t} a_{3}^{2}-a_{4} \bar{b}_{2}\right)+\bar{b}_{2}\left(-a_{3}^{2}+\left|b_{2}\right|^{2}\right)\right)+\bar{b}_{2}\left(-\left(\bar{t} b_{1}+t \bar{b}_{1}\right)\left|b_{2}\right|^{2}+a_{3}\left(|t|^{2}\left|b_{1}\right|^{2}+\left|b_{2}\right|^{2}\right)\right)}{\left(a_{1} a_{3}-\left|b_{1}\right|^{2}\right)\left(a_{2} a_{4}-\left|b_{2}\right|^{2}\right)}, \\
& \partial_{\bar{t}}\left(\frac{a_{2} \partial_{t} a_{4}-\bar{b}_{2} \partial_{t} b_{2}}{-a_{2} a_{4}+\left|b_{2}\right|^{2}}\right)=\frac{a_{2}\left(a_{4}^{2}\left(\bar{t} b_{1}+t \bar{b}_{1}\right)-a_{3}\left(a_{4}^{2}+|t|^{4}\left|b_{1}\right|^{2}\right)\right)}{\left(a_{1} a_{3}-\left|b_{1}\right|^{2}\right)\left(a_{2} a_{4}-\left|b_{2}\right|^{2}\right)} \\
& +\frac{\left(-a_{4}\left(\bar{t} b_{1}+t \bar{b}_{1}\right) b_{2}+a_{3}\left(|t|^{2} t\left|b_{1}\right|^{2}+a_{4} b_{2}\right)\right) \bar{b}_{2}+|t|^{2} a_{1}\left(a_{2}\left(|t|^{2} a_{3}^{2}-a_{4}^{2}\right)+\left(-t a_{3}^{2}+a_{4} b_{2}\right) \bar{b}_{3}\right)}{\left(a_{1} a_{3}-\left|b_{1}\right|^{2}\right)\left(a_{2} a_{4}-\left|b_{2}\right|^{2}\right)}
\end{aligned}
$$

substantiated by the constraints coming from reality condition

$$
\begin{aligned}
& \left(-\bar{t} a_{1}+\bar{b}_{1}\right)\left(-t a_{2}+b_{2}\right)+a_{1}\left(a_{4}-t \bar{b}_{2}\right)=1, \\
& a_{2}\left(a_{3}-t \bar{b}_{1}\right)+\left(-t a_{1}+b_{1}\right)\left(-\bar{t} a_{2}+\bar{b}_{2}\right)=1, \\
& a_{2}\left(a_{3}-\bar{t} b_{1}\right)+b_{1} \bar{b}_{2}=1, \\
& \bar{b}_{1} b_{2}+a_{1}\left(a_{4}-\bar{t} b_{2}\right)=1, \\
& a_{2} \bar{b}_{1}+a_{1}\left(-\bar{t} a_{2}+\bar{b}_{2}\right)=0, \\
& \left(a_{3}-\bar{t} b_{1}\right)\left(-t a_{2}+b_{2}\right)+\left(b_{1}\left(a_{4}-t \bar{b}_{2}\right)\right)=0, \\
& \left(a_{3}-t \bar{b}_{1}\right) b_{2}+\left(-t a_{1}+b_{1}\right)\left(a_{4}-\bar{t} b_{2}\right)=0 .
\end{aligned}
$$

The examination of this constrained and coupled non-linear system is out of the scope of this paper, although it should be a integrable one from the zero curvature structure of $t t^{*}$ equation.

\section{B. A sample residue calculation}

Here we give a sample calculation for the residue.

$$
K=\left\langle(x y)^{n-1} \mid(x y)^{n-1}\right\rangle=\int d x \wedge d y \frac{(x y)^{2(n-2)}}{\left(x^{n-1}-t y\right)\left(y^{n-1}-t x\right)}
$$

We evaluate the residue iteratively, that is, the vanishing of the denominator gives $y_{0 i}=$ $(t x)^{1 / n-1} \omega^{i}$ with $\omega^{n-1}=1$ and $y_{1}=x^{n-1} / t$. Then the residue integral of the $y$ becomes

$$
K=-\int d x \sum_{i=0}^{n-1} \frac{x^{2(n-2)} y_{0 i}^{2(n-2)}}{(n-1) y_{0 i}^{n-2}\left(x^{n-1}-t y_{0 i}\right)}+\int d x \frac{x^{2(n-2)} y_{1}^{2(n-2)}}{t\left(y_{1}^{n-1}-t x\right)}
$$




$$
=-\sum_{i=0}^{n-2} \frac{1}{n-1} \int d x \frac{x^{2(n-2)}(t x)^{(n-2) /(n-1)} \omega^{i(n-2)}}{x^{n-1}-t(t x)^{1 /(n-1)} \omega^{i}}+\frac{1}{t} \int d x \frac{\left(x^{n-1} / t\right)^{2(n-2)} x^{2(n-2)}}{\left(x^{n-1} / t\right)^{n-1}-t x}
$$

The first integral is zero since it can be brought to the polynomial denominator by change of variable $x=u^{n-1}$. But then the $u$ contour does not close the poles when $x$ wrap a circle. The second integral becomes

$$
\begin{aligned}
& (1 / t) \int d x \frac{t^{-2(n-2)} x^{2(n-2)(n-1+1)-1}}{t^{1-n} x^{n(n-2)}-t} \\
& =\left.(1 / t) t^{-2(n-2)} \sum_{i=0}^{n(n-2)-1} \frac{x^{2(n-2) n-1}}{t^{1-n} n(n-2) x^{n(n-2)-1}}\right|_{x=x_{i}} \\
& =t^{2}
\end{aligned}
$$

where $x_{i}$ is a solution of $x^{n(n-2)}=t^{n}$.

\section{Acknowledgement}

This work is supported by KOSEF Grant R01-2004-000-10520-0. 


\section{References}

[1] A. Adams, J. Polchinski and E. Silverstein, JHEP 10 (2001) 029.

[2] C. Vafa, "Mirror symmetry and closed string tachyon condensation," [arXiv:hep-th/0111051].

[3] J. Harvey, D. Kutasov, E. Martinec and G. Moore, "Localized tachyons and RG flows," [arXiv:hep-th/0111154].

[4] A. Dabholkar and C. Vafa, JHEP 0202 (2002) 008.

[5] A. Dabholkar, Phys. Rev. Lett. 88 (2002) 091301[arXiv:hep-th/0111004];

Soonkeon Nam and Sang-Jin Sin, J. Korean Phys. Soc. 43 (2003) 34 [arXiv:hep-th/0201132];

T. Sarkar, Nucl. Phys. B648 (2003) 497 [arXiv:hep-th/0206109];

Y. Michishita and P. Yi, Phys. Rev. D 65 (2002) 086006 [arXiv:hep-th/0111199];

T. Suyama, JHEP 0210 (2002) 051 [arXiv:hep-th/0210054];

D. R. Morrison and K. Narayan, JHEP 0502, 062 (2005) [arXiv:hep-th/0412337];

M. Headrick, S. Minwalla and T. Takayanagi, Class. Quant. Grav. 21, S1539 (2004) [arXiv:hep-th/0405064].

[6] A. Dabholkar, A. Iqubal and J. Raeymaekers, JHEP 0405, 051 (2004) [arXiv:hep-th/0403238];

Y. Okawa and B. Zwiebach, JHEP 0403, 056 (2004) [arXiv:hep-th/0403051];

G. W. Moore and A. Parnachev, JHEP 0411, 086 (2004) [arXiv:hep-th/0403016].

[7] Sang-Jin Sin, Nucl. Phys. B637 (2002) 395 [arXiv:hep-th/0202097];

Sang-Jin Sin, Nucl. Phys. B667 (2003) 310 [arXiv:hep-th/0308015];

Sang-Jin Sin, Phys.Lett. B578 (2004) 215 [arXiv:hep-th/0308028].

[8] Sunggeun Lee and Sang-Jin Sin, JHEP 0401 (2004) 054 [arXiv:hep-th/0312175].

[9] Sunggeun Lee and Sang-Jin Sin, Phys. Rev. D69 (2004) 026003 [arXiv:hep-th/0308029].

[10] Sunggeun Lee and Sang-Jin Sin, "Closed string tachyon potential and $t t^{*}$ equation," [arXiv:hep-th/0412247].

[11] S. Cecotti, P. Fendley, K. A. Intriligator and C. Vafa, Nucl. Phys. B386 (1992) 405 [arXiv:hep-th/9204102].

[12] K. Hori and C. Vafa,"Mirror symmetry," (unpublished) [arXiv:hep-th/0002222].

[13] E. Witten, Nucl. Phys. B403 (1993) 159 [arXiv:hep-th/9301042].

[14] S. Cecotti and C. Vafa, Nucl. Phys. B367 (1991) 359.

[15] N. Seiberg, Prog. Theor. Phys. Suppl. 102 (1990) 319. 